\title{
Androgen secreting ovarian tumors
}

Keywords: virilizing ovarian tumor, testosterone in female, sex cord stromal tumor

\section{Introduction}

Androgen secreting tumors arise from ovaries or adrenals. Virilizing ovarian tumors constitute less than $0.2 \%$ of cases of hyperandrogenism ${ }^{1}$ and less than $1 \%$ of all ovarian tumors. ${ }^{2}$ Androgen secreting tumors often present with rapidly progressive symptoms of hirsutism and virilization. There are some rare benign ovarian tumors where there is a slow progression and long duration of symptoms before the diagnosis is established. The histological classification of ovarian tumors categorization by the World Health Organization (WHO, (is based on their histogenetic principles, in regard to their derivation from the coelomic surface of epithelial cells, germ cells, and mesenchyma (the stroma and the sex cord). ${ }^{3}$ The sex cord-stromal and steroid cell tumors of the ovary are classified as sex cord-stromal cell tumor (granulosa and the coma-fibroma), sterol-stromal cell tumors, and mixed or unclassified and steroid cell tumors. Ovarian tumors that present with hyperandrogenism include Leydig cell tumors, Sertoli cell tumors, steroid cell tumors-not otherwise specified and ovarian thecomas. ${ }^{5}$

Other rare ovarian causes of hyperandrogenism include gynandroblastoma, gonadoblastoma, ovarian carcinoid, surface epithelial tumors (Brenner tumor) and metastatic tumors. ${ }^{5}$ These tumors are rare representing only $10 \%$ of all ovarian tumors. Tumoral cause of hyperandrogenism is suspected when the serum testosterone levels are significantly elevated $(>200 \mathrm{ng} / \mathrm{ml})$, rapidly progressive symptoms of virilization or pelvic mass in examination/imaging. As mentioned earlier, symptoms and signs associated with hillar Leydig cell tumor may present as slowly progressive hirsutism. ${ }^{6,7}$ When imaging does not identify ovarian or adrenal mass and the results of serum DHEAS are normal, then it is considered an indirect evidence of ovarian tumor if serum testosterone levels exceed 200ng/dl. These ovarian tumors secrete significant levels of testosterone or its precursor, and rostenedione.

The importance of ovarian cancer stems not from the fact that it is the sixth most common cancer in women worldwide, but that it is the most lethal gynecologic malignancy in Western Europe and North America, causing demise of more women than uterine and cervical cancer combined. It is predominantly a disease of postmenopausal women with a median age at diagnosis of 60 years. Both functional and nonfunctional ovarian neoplasms are known to produce androgen. Androgen-secreting neoplasms of the ovary are a rare cause of androgen excess and, account for only $5 \%$ of all ovarian tumors. Most are Sertoli-Leydig cell, lipid cell, theca cell or hilus cell tumors (Lobo, 1991). Granulosa cell tumors of the ovary are rare neoplasm (Mancaux et al., 2013), estrogen-producing tumors (Geetha and Nair, 2010) and difficult to diagnose (Mancaux et al., 2013).

It is important to identify and remove these tumors. Although clinical history and baseline androgen levels may increase clinical suspicion, localization of these tumors requires several diagnostic methods, including dynamic ovarian and adrenal assessment and diagnostic imaging (Taylor et al., 1986). However, sometimes they
Volume 5 Issue 6 - 2017

\author{
Neda Rouhi Brojeni,' Behrouz Salehian² \\ 'Department of Medicine, St.Vincent Charity Medical Center, \\ USA \\ ${ }^{2}$ Department of Diabetes, Endocrinology and Metabolism, City \\ of Hope and Beckman Research Institute, USA
}

Correspondence: Behrouz Salehian, City of Hope National Medical Center, 1500 East Duarte Road Duarte, CA 91010, USA, Tel 626 256-4673, Fax 62630I-8212 Email bsalehian@coh.org

Received: July 28, 2017 | Published: September 14, 2017

are very difficult to detect, and interpretation of test results can be difficult. Imaging modalities, such as magnetic resonance imaging (MRI), computerized tomography (CT) and ultrasound (US) might not identify ovarian lesions. ${ }^{8}$ Selective venous catheterization has been recommended as an accurate method for the diagnosis and localization of androgen-secreting ovarian and adrenal tumors (Lobo, 1991, Cohen et al., 1993), especially as most ovarian androgenproducing tumors are small (Lobo, 1991) and thus, difficult to detect by imaging techniques (Sarfati et al., 2011). However, Diagnostic specificity of this technique is often disappointing even in experienced hands (Sarfati et al., 2011). Moreover, in some cases, pre-operative venous sampling was attempted but not achieved, and thus venous sampling was done intra-operatively during diagnostic laparoscopy or explorative laparotomy (Kaltsas et al., 2003).

Sertoli Leydig cell tumor (Sertoli-stromal cell tumor, Arrhenoblastoma, Androblastoma). Sertoli-Leydig cell tumors (SLCT) are sex cord-stromal tumors that account for less than $0.5 \%$ of primary ovarian neoplasms. ${ }^{1}$ Because of their scarcity SLCT represent a challenge in diagnosis and management. The vast majority of SLCT (90\%) occur in the reproductive age and the rest before menarche or after menopause. ${ }^{2}$ They are usually unilateral and confined to the ovary at the time of diagnosis. Approximately $80 \%$ of SLCT are hormonally active with elevated serum testosterone and androstenedione levels and one third of these tumors is discovered due to the clinical signs and symptoms of excess androgen production (virilization, voice deepening, male pattern baldness, amenorrhea, clitoromegaly). ${ }^{3}$ Occasionally patients have estrogenic manifestations (menometrorrhagia, postmenopausal bleeding). ${ }^{9}$ Abdominal mass, pain and distension and ascites are the clinical presentations in approximately $50 \%$ of these tumors. ${ }^{4}$

Tumors that induce hyperandrogenicity may present with virilization signs (e.g., hirsutism, acne, irregular menstrual periods, male-pattern baldness, loss of female fat distribution, and hoarse voice), whereas tumor subtypes associated with abnormal estrogen production may present with hyperestrogenicity (e.g., isosexual precocity in children, abnormal uterine bleeding, endometrial hyperplasia, and carcinoma). SLCT is the most common virilizing ovarian tumor, as approximately $30 \%-50 \%$ of these tumors produce androgens (testosterone and androgen precursors) and more than one-third of cases develop 
symptoms of virilization $(4,15,18)$. Notwithstanding, SLCT is a rare cause of virilization in premenopausal women. In this age group, other differential diagnosis such as polycystic ovary syndrome and adrenal androgen-secreting tumors should be considered. In cases of androgen-secreting SLCTs, the laboratory data normally indicate elevated serum testosterone levels but, in contrast to masculinizing adrenal tumors, normal or slightly elevated urinary 17-ketosteroid levels (4).

Many SLCTs are hormonally inactive and a small subgroup is estrogenic. Sudden abdominal pain and swelling are frequent symptoms of nonfunctioning tumors. According to the WHO classification ${ }^{5}$ SLCTs are subdivided into well, moderately and poorly differentiated tumors depending on the degree of tubular differentiation of the Sertoli cell component (with poorly differentiated tumors having the least tubular differentiation). Heterologous mesenchymal or epithelial elements are found in up to $20 \%$ of SLCTs and they occur only in moderately and poorly differentiated tumors or tumors with retiform pattern. ${ }^{6}$ The prognosis depends on the patient's age, stage of the tumor and the degree of differentiation with the presence of heterologous elements or retiform pattern being a bad prognostic feature. ${ }^{1}$ SLCTs of the ovary are classified into 3 grades according to their level of differentiation:

a. Grade 1, well-differentiated;

b. Grade 2, moderately-differentiated; and

c. Grade 3, poorly-differentiated (9).

SLCTs have been reported to most commonly occur unilaterally (1, 10 , and 11). The tumor may present as solid, cystic or a combination of the two (1). The prognosis of SLCTs is overall favorable and depends on the stage and histological grade of the tumor and the age of the patient. The overall 5-year survival rate for stage I is $95 \%$ and for stages III and IV almost $0 \%$. SLCTs may produce androgens or estrogens, and their prognosis is associated with tumor differentiation and disease stage (1). The diagnosis of an SLCT of the ovary may require considerable time and effort. SLCTs may consist of testicular elements and produce androgens or estrogens (1). Due to androgen secretion, certain SLCT patients may present with masculinization, with symptoms that include anovulation, amenorrhea, defeminization, hirsutism, cliteromegaly, laryngeal protuberance and voice raucity (10). The presence of an ovarian tumor with clinical signs of hormonal disturbances, particularly in younger women, should alert clinicians to the potential presence of an ovarian SLCT. The time interval between the oversupply of androgen secretion and the diagnosis of SLCT typically varies from 6-9months (1).

Very rarely, SLCT have been reported to associate with elevated alpha-fetoprotein serum levels; this is a typical feature of germ cell tumors such as yolk sac neoplasm. Although only approximately 30 cases have been described to date, SLCT is the most commonly reported ovarian nongerm cell tumor associated with elevated serum alpha-fetoprotein levels. In certain cases the tumor may be accidentally found during a routine gynecological ultrasound, when suspicious abdominal masses may be observed. If the mass is large it may cause abdominal pain and distension. In addition, rupture of the mass with acute abdominal pain is possible (10). CT and magnetic resonance imaging have made a poor contribution to the diagnosis of SLCTs; $20 \%$ of SLCTs are small in size, and thus unidentifiable, rendering their accurate diagnosis and localization challenging (10). Furthermore, SLCTs are able to coexist with alternative types of ovarian tumors, resulting in patients presenting with mixed symptoms, making diagnosis more challenging. Previous studies have demonstrated that SLCT is associated with mucinous adenocarcinoma and a Brenner tumor of the ovary $(15,16)$. SLCT has a nonspecific appearance. On US, these tumors usually present either as a distinct hypoechoic mass or a heterogeneous mass that is primarily solid with multiple cystic spaces. Small virilizing SLCTs may be easily detected using color Doppler US rather than transvaginal US alone (13, 45, and 46). On CT images, a soft-tissue attenuating adnexal mass is usually seen (15). The solid tumor portions characteristically exhibit avid contrast uptake.

On MRI, the T2 signals of the solid components differ according to the extent of fibrous components. Nonetheless, strong hypointensity on T2-weighted images is not characteristic $(13,18)$ (Fig. 10). Hypointense areas on T1-weighted images and hyperintense areas on T2-weighted images reflect cystic areas. Cysts may also exhibit mild hyperintensity on T1-weighted images (47). Striking homogeneous or heterogeneous contrast enhancement of the solid areas is observed on gadolinium-enhanced images. SLCTs are most commonly diagnosed in young women, creating an issue with regard to the preservation of fertility (3). Oncologists should consider the risk of infertility in women of reproductive age who undergo treatment for SLCTs. Patients must be thoroughly informed about the conservative treatment alternatives for genital tract preservation, as well as the potential risk of recurrent disease. The conservative management consists of unilateral salpingo-oophorectomy, usually accompanied by biopsy of the contralateral ovary. Hysterectomy and bilateral salpingo-oophorectomy comprise the radical treatment strategies (4). Well-differentiated SLCTs typically present with benign behavior (10). Malignancy is observed in $11 \%$ of moderately-differentiated SLCTs (10-year survival rate, $87 \%$ ) and $59 \%$ of poorly-differentiated SLCTs (10-year survival rate, $41 \%$ ). Furthermore, the 5-year overall survival rate in cases of stage I disease is almost $92 \%$ (17).

Because SLCTs are very rare, a standardized approach for treatment has not been established yet. The primary treatment for SLCTs is surgery, based on the patient age, disease stage and tumor grade $(6,9)$. However, a significant issue arising from disease occurrence in patients of reproductive age is the preservation of fertility (afforded by a cystectomy/adnexectomy with preservation of the rest of the female genital tract). Hysterectomy with bilateral salpingo-oophorectomy, as well as staging surgery (cytology, omentectomy, pelvic-paraaortic lymphadenectomy or peritoneal biopsies), is the optimal approach for patients beyond childbearing age, although the necessity of a lymphadenectomy remains controversial (17). Chemotherapy may be administered following surgical treatment, particularly for tumors demonstrating poor or moderate differentiation (10). According to Gui et al (10), adjuvant chemotherapy does not provide any significant advantages, and it should be considered primarily in cases of poorly- and moderately-differentiated tumors or disease recurrence. Additionally, a previous study (MITO) was able to demonstrate the benefit of the administration of post-operative chemotherapy for patients exhibiting advanced stage (II-IV) disease (17).

\section{DICER I syndrome}

Little is known about the pathogenesis of ovarian SLCT however, germ-line mutations in the microRNA processing gene DICER1 have been shown to be related with the development of benign tumors that are susceptible to malignant transformation. ${ }^{10}$ These tumors include 
ovarian SLCT, multinodular goiter, multilocular cystic nephroma and pleuropulmonary blastoma. ${ }^{11-13}$ Heravi-Moussavi et al., ${ }^{11}$ report a $60 \%$ prevalence of DICER1 mutations in SLCT. Similarly, in the study of Conlon et al. ${ }^{14}$ DICER1 mutations were present in $63 \%$ of SLCT. They also report that there is no association between DICER1 mutation and tumor differentiation as mutations in poorly differentiated and moderately differentiated SLCT were found to have similar frequencies. It has been established, that reduced expression of DICER1 is associated with a poor cancer outcome. The exact mechanism underlying tumorigenesis associated with DICER1 mutations remains to be determined. ${ }^{15}$

\section{Steroid cell tumor}

They are grouped under sex-chord stromal tumors and are usually benign, unilateral and characterized by a steroid cell proliferation. Historically these tumors have been variously referred to as lipoid cell tumors, lipid cell tumors, adrenal-like tumors, masculinovoblastomas, leutomas, hypernephroid tumors, and adrenal rest tumors, indicating the infidelity of their supposed lineage. Ovarian steroid cell tumors are rare functioning sex cord-stromal tumors. The average age at diagnosis is the mid-20s, but patients can present at virtually any age. These tumors are composed entirely or predominantly of cells that resemble steroid hormone secreting cells and are categorized according to histologic composition of these cells. ${ }^{1}$ Ovarian Steroid Cell tumor can be sub-classified into Stromal luteoma, Leydig cell tumors and steroid cell tumors not otherwise specified Sex-cord stromal tumors. Steroid cell tumors account for $0.1 \%$ of all ovarian tumors and the subtype not otherwise specified (NOS) are the most common subtype within this group and the majority of tumors occur in pre-menopausal women (mean age: 43years) in which $56-77 \%$ of patients present with virilization due to excess testosterone. Clinical manifestations are defined by the type of hormone produced by steroid cell tumors, NOS. Most secrete testosterone (56 to 77\%) and patients present with virilizing symptoms such as gradually progressive hirsutism, acne, deepening of voice, temporal baldness and amenorrhea.

Hyperestrogenemia presenting as menorrhagia or irregular uterine bleeding has been reported in 6 to $23 \%$ of women. 1 in 6 to $10 \%$ of cases, excess cortisol secretion can lead to Cushing syndrome. However, $25 \%$ of patients with steroid cell tumor NOS are asymptomatic. They are further divided into three subtypes: stromal leuteoma, Leydig cell tumor, and steroid cell tumor, ${ }^{1,3,9}$ the latter being the most common of the three subtypes accounting for approximately $60 \%$ of cases. Steroid cell tumors are associated with androgenic changes with variable frequency, ranging from $12 \%$ to over $50 \%$, and they are usually of many years duration.

\section{Granulosa cell tumor of the ovary}

Granulosa cell tumors of the ovary (GCT), the major form of ovarian stromal tumors, arise from proliferating granulosa cells of the ovarian follicle. They exhibit features of granulosa cells that include estrogen biosynthesis as well as the production of gonadal peptides including inhibin and anti-Müllerian hormone (AMH). GCT is classified as adult ( $95 \%$ of GCT) or juvenile, based on histopathological and clinical criteria. Adult granulosa cell tumors (AGCTs) account for less than $5 \%$ of all ovarian malignancies, whereas the majority (95\%) occurs after the age of 30 (adult-type) and present at an early stage. AGCT of the ovary is oftentimes a hormonally active stromal cell neoplasm that is distinguished by its ability to express aromatase and to secrete sex steroids such as estrogen. Ovarian granulosa cell tumors are rare hormonally active neoplasms accounting for $\square 3-5 \%$ of all ovarian cancers (2). Juvenile granulosa cell tumors (JGCTs) of the ovary are more aggressive than the adult type, but also far less common, representing only $5 \%$ of all granulosa cell tumors in the paediatric age group (3) (4). Granulosa cell tumors secrete steroid hormonesmostly estrogens and less commonly androgens and progestogens (2), but secretion of prolactin (5) and insulin (6) has been described. Most granulosa cell tumors secrete inhibin which has been used as granulosa cell-specific tumor marker (7). Most JGCTs are FIGO stage 1 at the time of diagnosis and have excellent cure rates (5-year survival rate $90-95 \%$ ) with surgery being the mainstay of therapy. Advanced stages bear a worse prognosis (5-year survival rate $25-50 \%$ ) and require adjuvant chemotherapy with cisplatin-based regimens (8).

\section{Thecoma}

Ovarian thecoma is a rare benign tumor of stromal cell origin, and represents $<1 \%$ of all ovarian tumors (1). They occur mostly in postmenopausal women, with a mean age of 59years, and only $10 \%$ of the patients are normally $<30$-years-old. These tumors had presented as primary amenorrhea, secondary amenorrhea, hirsutism, menstrual irregularity or pressure effects. Ovarian thecoma with virilizing manifestations were reported in both post-menopausal and premenopausal age groups. Ovarian thecoma with concomitant post-menopausal bleeding, endometrial hyperplasia and endometrial carcinoma were reported in a post-menopausal woman. Thecomas range from small tumors to large solid or solid-cystic masses of up to $15 \mathrm{~cm}$. They are unilateral in $>90 \%$ of cases and are rarely malignant $(2,3)$. Thecomas are stromal tumors made up of cells that resemble theca cells, lutein cells and fibroblasts (4). They are traditionally classified within the sex cord-stromal tumor category of ovarian tumor types (5).

\section{Sclerosing stromal ovarian tumor}

Sclerosing tumors account for $2 \%$ to $6 \%$ of ovarian tumors originating from stroma of ovary and account for $1.5 \%$ of all ovarian tumors (1). More than $80 \%$ of sclerosing stromal tumors usually occur in the second and third decades of life (mean age, 21years). The most common presenting symptoms are menstrual irregularity, abdominal pain and a lower abdominal mass. Sclerosing stromal tumors are usually hormonally inactive. If hormonally active, they are usually androgenic and occur most frequently during pregnancy. Menstrual irregularity and pelvic pain are the most frequent complaints during presentation.

The etiology of sclerosing stromal tumors is unknown. The gross appearance of sclerosing stromal tumors of ovary ranges from small solid masses to large multicystic masses. The important differential diagnoses include other sex cord-stromal tumors including fibroma, thecoma, lipid cell tumor, vascular tumors, malignant tumors such as Krukenberg's tumor, and non-neoplastic conditions such as massive odema of ovary. Calretinin, inhibin, CD34, and $\alpha$-glutathione S-transferase $(\alpha-G S T)$ positivity has been reported to be useful in differentiating sclerosing stromal tumors from other sex cord-stromal tumors. Sclerosing stromal tumor is an extremely rare and distinctive sex cord-stromal tumor, occurring predominantly in the second and third decades of life. Due to the rarity of this neoplasm it is not always possible to predict the presence of this tumor preoperatively on the basis of clinical and radiological findings. The possibility of sclerosing 
stromal tumor should always be kept in young patients with ovarian mass, as these benign and can be treated successfully by enucleation or unilateral ovariotomy.

\section{Ovarian tumors with functioning stroma}

Hormonally active ovarian tumors, such as granulosa cell tumors, thecomas, and Sertoli-Leydig cell tumors, are classified as sex cordstromal or steroid cell tumors. In several reports, elevated serum levels of steroid hormones including E2 were noted in postmenopausal women with common epithelial ovarian tumors. Both malignant and benign ovarian tumors produce E2. Several studies have reported relatively high peripheral E2 $(>20 \mathrm{pg} / \mathrm{ml})$ concentrations in about $50 \%$ of postmenopausal women with ovarian epithelial tumors, compared to the levels observed in postmenopausal women without ovarian tumors. ${ }^{1-3,9}$ Among them, the E2 released from ovarian tumors has biological effects on bone metabolism and increases the incidence of abnormal vaginal bleeding or abnormally thick endometrium.

A high serum estradiol (E2) level is occasionally detected in postmenopausal women with common epithelial ovarian tumors with functioning stroma. It has been proven that functioning stroma has the capacity to convert androgens to estrogens. Such ovarian tumor stroma exhibiting lutein-like or theca-like cells, or both, is frequently detected with morphological studies and has been termed "ovarian tumors with functioning stroma". Ovarian tumors with functioning stroma, regardless of cell morphology, have a capacity for converting androgens (mainly of adrenal origin) to estrogens, but this conversion usually takes place in peripheral tissues.

\section{Acknowledgements}

None.

\section{Conflict of interest}

The author declares no conflict of interest.

\section{References}

1. Cohen I, Nabriski D, Fishman A. Noninvasive test for the diagnosis of ovarian hormone-secreting-neoplasm in postmenopausal women. Gynecol Oncol Rep. 2016;15:12-15.

2. Burnik Papler T, Frković Grazio S, et al. Sertoli-Leydig cell tumor with retiform areas and overgrowth of rhabdomyosarcomatous elements: case report and literature review. J Ovarian Res. 2016;9(1):46.
3. Stavrakis T, Kalogiannidis I, Petousis S, et al. Fertility-sparing management and obstetric outcomes in a 20-year-old patient with a Sertoli-Leydig cell tumor of the ovary: A case report and review of the literature. Oncol Lett. 2016;12(2):1079-1082.

4. Ting Y, Yang LI, Juan Z, et al. Ovarian thecoma with massive pleural effusion in postmenopausal women: A case report. Mol Clin Oncol. 2015;4(6):1003-1005.

5. Park JW, Bae JW. Postmenopausal Meigs' Syndrome in elevated CA-125: a case report. J Menopausal Med. 2015;21(1):56-59.

6. Ramkumar S, Jyotsna VP, Mallick S, et al. Bilateral thecoma presenting as premenopausal hirsutism: Laproscopic removal. Indian J Endocrinol Metab. 2013;17(Suppl 3):S662-664.

7. Atram M, Anshu, Sharma S, Gangane N. Sclerosing stromal tumor of the ovary. Obstet Gynecol Sci. 2014;57(5):405-408.

8. Tian T, Zhu Q, Chen W, et al. CT findings of sclerosing stromal tumor of the ovary: A report of two cases and review of the literature. Oncol Lett. 2016;11(6):3817-3820.

9. Horta M, Cunha TM. Sex cord-stromal tumors of the ovary: a comprehensive review and update for radiologists. Diagn Interv Radiol. 2015;21(4):277-286.

10. Sood N, Desai K, Chindris A-M, et al. Symptomatic ovarian steroid cell tumor not otherwise specified in a post-menopausal woman. Rare Tumors. 2016;8(2):6200.

11. Bettegowda A, Rangaiah N, Prasad N, at al. Virilizing ovarian steroid cell tumor: a rare case. J Clin Diagn Res. 2015;9(9):QD05-QD06.

12. Iqbal A, Novodvorsky P, Lubina-Solomon A, et al. Juvenile granulosa cell tumour of the ovary presenting with hyperprolactinaemic amenorrhoea and galactorrhoea. Endocrinol Diabetes Metab Case Rep. 2016;2016:160006.

13. Vasu PP, Leelamma JP, Mohammed BA, et al. Primary granulosa cell tumor of retroperitoneal origin: A rare presentation with emphasis on cytomorphology. J Cytol. 2016;33(1):52-54.

14. Alexiadis M, Chu S, Leung D, et al. Transcriptomic analysis of stage 1 versus advanced adult granulosa cell tumors. Oncotarget. 2016;7(12):14207-14219.

15. Hattori Y, Yamada S, Yamamoto M, et al. Ovar an mucinous adenocarcinoma with functioning stroma in postmenopausal women: aromatase and SF-1 expressions. J Ovarian Res. 2015;8:73. 